\title{
HopScotch-a low-power renewable energy base station network for rural broadband access
}

\author{
Colin McGuire ${ }^{1 *}$, Malcolm R Brew², Faisal Darbari ${ }^{1}$, Gregour Bolton ${ }^{1}$, Anthony McMahon ${ }^{2}$, David H Crawford ${ }^{1}$, \\ Stephan Weiss ${ }^{1}$ and Robert W Stewart ${ }^{1}$
}

\begin{abstract}
The provision of adequate broadband access to communities in sparsely populated rural areas has in the past been severely restricted. In this article, we present a wireless broadband access testbed running in the Scottish Highlands and Islands which is based on a relay network of low-power base stations. Base stations are powered by a combination of renewable sources creating a low cost and scalable solution suitable for community ownership. The use of the $5 \mathrm{GHz}$ bands allows the network to offer large data rates and the testing of ultra high frequency "white space" bands provides expansive coverage whilst reducing the number of base stations or required transmission power. We argue that the reliance on renewable power and the intelligent use of frequency bands makes this approach an economic green radio technology which can address the problem of rural broadband access.
\end{abstract}

\section{Introduction}

Broadband internet access is regarded as a key enabling technology in both developed and developing economies. Widespread broadband internet access in developed economies such as the UK and US has led to a move towards online services such as e-government, telemedicine, video conferencing, and video streaming. Despite this proliferation, a digital divide has emerged between urban and rural communities [1] as telecommunication providers are unable to roll out broadband internet access to remote, sparsely populated rural communities due to the large costs involved and poor return on investment.

In remote areas such as the sparsely populated Highlands and Islands of Scotland many households are located further than $4 \mathrm{~km}$ from an exchange, which limits digital subscriber line (DSL) broadband connectivity with the existing copper cabling. Emerging alternatives such as fibre to the home and 3rd generation (3G) mobile broadband require substantial capital investment, planning permission and access to the electricity grid or the use of diesel generators to ensure uninterrupted

\footnotetext{
* Correspondence: cmcguire@eee.strath.ac.uk

${ }^{1}$ Centre for White Space Communications, Department of Electronic \& Electrical Engineering, University of Strathclyde, Glasgow G1 1XW, Scotland, UK

Full list of author information is available at the end of the article
}

power supplies. The large infrastructure costs and the additional operating expense of electrical connection and/or fuel make these standard solutions that have been adopted in urban settings uneconomical for rural deployment without major subsidy.

This article reports on the HopScotch project running in Scotland which at its core features the deployment of a green low power solution for rural broadband access. To motivate our study, this introductory section will review related efforts in wireless system development for rural locations and emphasise the green aspects of the HopScotch project and its associated "WindFi" renewable energy base stations which ensure the applicability and successful deployment of HopScotch from both an environmental and economic perspective.

\subsection{Rural communications and broadband access}

Digital service provision to rural communities around the world has been recognised as a problem for some time and has been addressed in [1,2]. Initial solutions have been fairly simple and limited, such as the idea of "one phone per village" [3]. Other systems, such as the relay-based DART deployed in rural Africa [4], have offered only very limited data communications in addition to voice.

Rural broadband access has been addressed more recently in a number of publications, generally favouring 
wireless links over optical fibre and satellite communications due to economic reasons, and over telephone copper wire due to limited bandwidth once distances from the telephone exchange grow longer [5]. Nevertheless, optical cable solutions are occasionally viable and given the large connectivity bandwidth created, can be considered as "low cost" [6]. In [7], a cost comparison for Croatian villages is made between wireline (DSL) and wireless (WiMAX) access, favouring the former, whereby the considered density is close to the 150 inhabitants $/ \mathrm{km}^{2}$ which the UN has defined as an upper limit to what can be defined as a rural setting. Similar wireless links have been evaluated in Montana using line of sight (LOS) conditions for rural broadband access [8]. A similar system based on WiMAX is outlined in [9], where a WiMAX rural access is connected via point-topoint (P2P) connection to the IP-based backbone. In [10], an argument is made for the opportunistic access to very high frequency (VHF) and ultra high frequency (UHF) bands, where propagation is more benign and not restricted to LOS conditions.

In terms of data throughput, the LOS-based system in [8] reports $11 \mathrm{Mbit} / \mathrm{s}$ over $4.5 \mathrm{~km}$ LOS or a lower throughput of $1 \mathrm{Mbit} / \mathrm{s}$ over a longer distance of 7.2 $\mathrm{km}$. Mosenthal et al. [5] studied fixed wireless links with a throughput up to $1.5 \mathrm{Mbit} / \mathrm{s}$, while WiMAX-based systems such as [9] are restricted to below $4 \mathrm{Mbit} / \mathrm{s}$.

Other studies have focused on creating low power rural networks in order to use renewable energy sources to power network nodes either entirely or as a supplement to existing energy sources. The Tegola IEEE 802.11 based mesh network exploits the inverse relationship between wind speed and solar irradiation throughout the year in Scotland to power nodes with a combination of wind and solar sources [11]. WiFi based rural networks in particular have become widespread, especially in developing countries, due to the low cost and power requirements of WiFi chipsets. In climates where solar radiation is in abundance all year round, solar power has been used effectively to either supplement existing energy sources or serve as the primary energy source for rural wireless mesh networks $[12,13]$. In the Digital Gangetic Plains network in India, the technical feasibility of establishing long-distance WiFi links is explored [14], where batteries charged by photovoltaic (PV) modules are used as backup due to unreliable mains power access. The need to optimise the power consumption of access points and routers for rural applications is widely acknowledged [15] and is the focus of continued research [16].

\subsection{Green radio requirements for wireless rural broadband access}

Green radio research covers a wide range of areas, with the collective goal of reducing energy requirements in radio systems. Studies into front end efficiency [17], savings in the network layer $[18,19]$, transmission protocols 20 and radio resource management [21] all contribute to energy efficiency.

Cognitive radio (CR) devices can adapt to varying conditions to fulfill green radio criteria by optimising power and spectrum efficiency based on an awareness of surroundings [22]. Dynamic spectrum access allows transmission power to be minimised by selecting the optimum frequency and bandwidth to minimise propagation losses [23]. Energy consumption can also be scaled in relation to throughput requirements and available bandwidth by modifying the coding rate and modulation scheme [24].

Within a radio, the power amplifier is generally considered the most power-hungry component in the transmitter [25] and reducing the transmit power is therefore the first target when aiming to reduce power consumption. Other components, such as the processing, have also been addressed, and savings can be achieved by scaling the processor clock according to throughput [26].

The use of low power devices, strategies and network designs facilitate the use of renewable energy sources. Remote regions where grid supplied power is either unavailable or unreliable often utilise diesel generators to provide power. In addition to emitting $\mathrm{CO}_{2}$, diesel generators can be costly to run due to ever increasing fuel costs and the added expense of fuel transportation to remote areas. Renewable energy sources such as wind turbines and PV arrays are an attractive alternative for remote telecommunications installations both environmentally and economically. An off-grid renewable power system allows a base station to be placed in the optimum location to serve users, requires no infrastructure to connect to an existing power source and, as no fuel is required, has minimal operating costs. Therefore, using a renewable power system provides an economic as well as environmental justification for the use of low power and energy efficient radios, systems and network designs. As the energy generation and storage system represents a significant proportion of the total cost of a renewable powered base station, minimizing the overall power consumption reduces the required generation potential and storage capacity.

The emergence of green radio technologies and initiatives allow wireless networks to be an economical and environmentally responsible rural broadband solution. Low power radios, systems and network design, CR and off-grid renewable energy sources can be combined to create an affordable yet powerful wireless network capable of delivering high bandwidth content and services.

\subsection{Contribution and overview of article}

In this article, we report on a rural broadband network termed HopScotch. Test installations of HopScotch base 
stations using $5 \mathrm{GHz} \mathrm{WiFi}$ and UHF "white space" radios are currently being trialled in Scotland. Communities are illuminated using a point-to-multipoint (PTMP) wireless distribution system connected to an IP-backbone directly or via a series of point-to-point (PTP) relays mounted on HopScotch renewable energy base stations or elsewhere if electrical power is easily available.

In line with the arguments in Section 1.2, HopScotch base stations are set up to be low power autonomous units, powered by a combination of renewable sources. One form of license-free network deployable today uses standard WiFi access technology similar to systems reviewed in Section 1.1 to set up a LOS network. The project also features the use of UHF "whitespace" radios thus forming a digital dividend white space spectrum overlay in the $600-800 \mathrm{MHz}$ band [27]. Hence by setting up a WiFi based network in a community we can easily overlay a whitespace test network. This will aid the study and comparison of access technologies, serve as a testbed for the investigation and development of TV white space (TVWS) links which have the potential to provide more comprehensive cover than existing fixed wireless bands in a rural setting, and further the potential of flexibility offered by designing and reviewing cognitive radio techniques.

This article is organised as follows. Section 2 introduces the idea of the HopScotch renewable energy network and discusses the benefits and challenges of the network communicating over both the $5 \mathrm{GHz}$ and "white space" spectrum. The concept of HopScotch is based on a network of "WindFi" base stations, which are described in Section 3 with a particular focus on the green technologies used to reduce the power consumption and cost of these devices. With respect to the transmission over either $5 \mathrm{GHz}$ or UHF bands, Section 4 analyses how frequency selection impacts on the network and its green radio credentials. Finally, conclusions will be drawn in Section 5.

\section{HopScotch network \& testbed}

Figure 1 shows how HopScotch could typically connect a community to the IP-backbone, with PTP links creating a network backbone between "WindFi" base stations, and PTMP links illuminating the community.

HopScotch uses standard IEEE 802.11n, operating in the $5 \mathrm{GHz}$ spectrum for PTP links and serving subscribers in close vicinity of the base station. The infrastructure can additionally feature an overlay "white space" network/testbed in a licensed UHF band or for that matter any other frequencies which are unused in rural areas. By using a combination of spectral bands this will allow for an optimum trade-off between channel throughput and coverage for different scenarios. The use of licensed and unlicensed spectrum in the $5 \mathrm{GHz}$ band allows off-the-shelf WiFi equipment to use a large

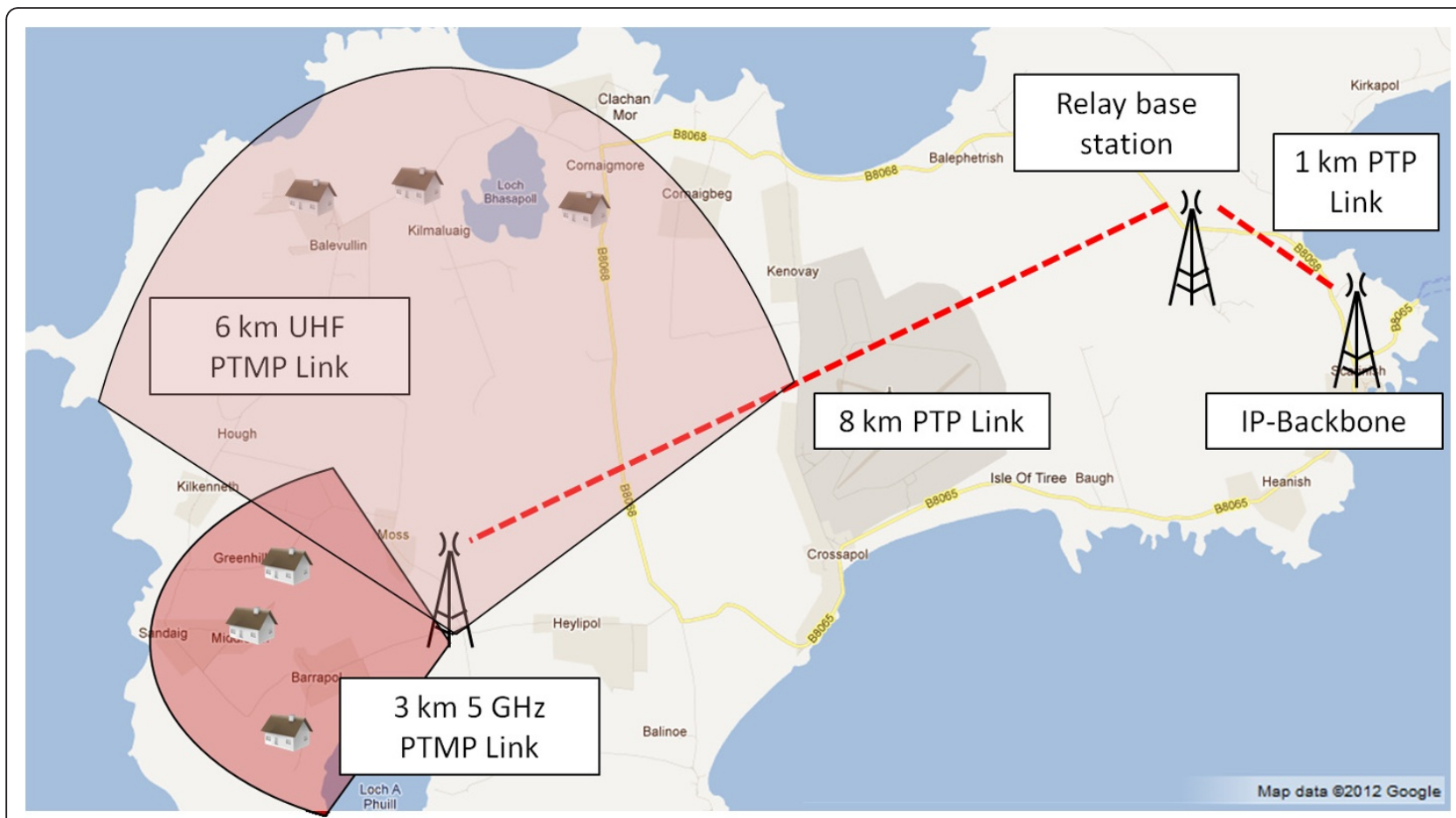

Figure 1 Example of a HopScotch network connecting a remote community to IP-backbone. 
channel bandwidth providing high throughput. The use of UHF frequencies will allow for greater coverage from the base station, especially in challenging radio terrain as detailed in Section 4, at the expense of a limited channel bandwidth and throughput.

\subsection{WiFi wireless LAN spectrum}

Three frequency bands are available for outdoor use based on off-the-shelf IEEE 802.11 abgn WiFi equipment in the UK, as shown in Table 1 . The $5 \mathrm{GHz}$ bands $B$ and $C$ are attractive for fixed rural broadband due to the relaxed transmit power limitations compared to 2.4 $\mathrm{GHz}$. Band B is lightly licensed to allow a greater transmit power for fixed wireless links.

\subsection{TV white space spectrum}

The TV spectrum in Europe is divided into $8 \mathrm{MHz}$ wide channels, ranging from $470 \mathrm{MHz}$ (channel 21) to 862 $\mathrm{MHz}$ (channel 69). When in 2012 the UK's last analogue television signals will be switched over to digital terrestrial TV (DTT), $112 \mathrm{MHz}$ of this band will remain vacant, freeing 14. UHF channels as shown in Figure 2. Channels 36 and 69 will also be released. Secondary reuse of interleaved bandwidths within the DTT range will be allowed by the UK's office for communications (Ofcom) as long as this will not interfere with a primary, licensed transmitter. The released spectrum together with any interleaved bandwidths available within the DTT range is referred to as "white space". The availability of this spectrum depends on the geographical location and is generally higher in rural areas.

In the UK the available TVWS frequencies are likely to range between $470 \mathrm{MHz}$ and $790 \mathrm{MHz}$ with $8 \mathrm{MHz}$ wide channels, for which Ofcom is currently formulating a policy for future use. In the US, the Federal Communications Commission (FCC) has already ruled on the use of TVWS frequencies in the US covering $54 \mathrm{MHz}$ to $692 \mathrm{MHz}$ with $6 \mathrm{MHz}$ channels. For fixed devices the maximum allowed equivalent isotropically radiated power (EIRP) is $4 \mathrm{~W}$ in channels 2 to 51 (excluding 3, 4 , and 37) [28].

Table $15 \mathrm{GHz}$ and $2.4 \mathrm{GHz}$ spectrum and equivalent isotropically radiated power (EIRP) limitations in the UK for outdoor use [source: Ofcom]

\begin{tabular}{llll}
\hline Band & $\mathbf{5 ~ G H z ~ B a n d ~ B ~}$ & $\mathbf{5 ~ G H z}$ Band C & $\mathbf{2 . 4} \mathbf{~ G H z}$ \\
\hline Frequency Range & $5470-5725 \mathrm{MHz}$ & $5725-5850 \mathrm{MHz}$ & $2400-2483.5 \mathrm{MHz}$ \\
Bandwidth & $255 \mathrm{MHz}$ & $125 \mathrm{MHz}$ & $83.5 \mathrm{MHz}$ \\
$20 \mathrm{MHz}$ Channels & 11 & 5 & 4 \\
$40 \mathrm{MHz}$ Channels & 6 & 2 & 2 \\
License & License exempt & Lightly licensed & License exempt \\
Maximum EIRP & $1 \mathrm{~W}$ & $4 \mathrm{~W}$ & $100 \mathrm{~mW}$ \\
\hline
\end{tabular}

\subsection{Network architecture}

An example HopScotch network serving multiple communities is shown in Figure 3. IP-backbone access to the island is used to provide connectivity to the ring network. A number of communities are then illuminated using $5 \mathrm{GHz}$ and UHF PTMP links both mounted on a HopScotch base station. A number of $5 \mathrm{GHz}$ links provide LOS access up to $3 \mathrm{~km}$ and UHF links provide non-LOS coverage up to $6 \mathrm{~km}$. "WindFi" base stations are connected together using $5 \mathrm{GHz}$ PTP links to form a ring between the two access points. This creates redundancy in the network, as each base station has two routes to backbone access. In the case of one HopScotch relay base station being shut down, IP access for other nodes is maintained as one path to the backbone will still be available. Spurs from the main loop can connect additional communities as required.

The core of the HopScotch network is based on the IEEE 802.11n standard which exploits techniques such as spatial multiplexing, channel bonding and frame aggregation to maximise throughput. A proprietary time division multiple access (TDMA) medium access controller (MAC) further improves the net throughput of the system over long distances.

PTP links are created using the $5 \mathrm{GHz}$ lightly licensed band C (5.725-5.850 GHz) with maximum EIRP of $4 \mathrm{~W}$. This $125 \mathrm{MHz}$ spectrum is divided into two non-overlapping $40 \mathrm{MHz}$ wide turbo channels, where each channel supports spatial multiplexing (i.e., $2 \times 2 \mathrm{MIMO}$ streams on vertical and horizontal polarisations). The resulting system with two independent spatial streams supports a theoretical data rate of $300 \mathrm{Mbit} / \mathrm{s}$.

PTMP links use the unlicensed band B (5.470-5.725 $\mathrm{GHz}$ ) with a maximum EIRP of $1 \mathrm{~W}$. The $255 \mathrm{MHz}$ wide spectrum is divided into 11 non-overlapping 20 $\mathrm{MHz}$ or six $40 \mathrm{MHz}$ channels. This allows a base station to use a flexible number of sectors to serve a community and provide omni-direction coverage around the base station. Initial estimates suggest that due to the sparsity of the rural population, each sector may serve a community of 8 to 10 broadband users on a theoretical $65 \mathrm{Mbit} / \mathrm{s}$ wireless link, therefore the number of sectors and hence the bandwidth used for each sector can be scaled as required.

UHF links are primarily limited to a $5 \mathrm{MHz}$ bandwidth to to fit within a TV channel. It is possible to concatenate adjacent $8 \mathrm{MHz}$ channels depending on their availability within a specific region. This could provide additional channel bandwidth thus resulting in even higher throughput.

\subsection{Current trials}

Green base station trials have been running in Scotland over the last 24 months, including a $10 \mathrm{~m}$ high hybrid 


\begin{tabular}{|c|c|c|c|c|c|c|c|c|c|c|c|c|}
\hline \multirow{11}{*}{$\begin{array}{l}\text { Channel } \\
\text { Frequency } \\
\text { (MHz) }\end{array}$} & 21 & 22 & 23 & 24 & 25 & 26 & 27 & 28 & 29 & 30 & 31 & 32 \\
\hline & $470-478$ & $478-486$ & $486-494$ & 494.502 & $502-510$ & 510-518 & $518-526$ & $526-534$ & $534-542$ & $542-550$ & 550-558 & $558-566$ \\
\hline & 33 & 34 & 35 & 36 & 37 & 38 & 39 & 40 & 41 & 42 & 43 & 44 \\
\hline & $566-574$ & 574-582 & $582-590$ & 590-598 & $598-606$ & 606-614 & $614-622$ & $622-630$ & 630-638 & $638-646$ & $646-654$ & $654-662$ \\
\hline & 45 & 46 & 47 & 48 & 49 & 50 & 51 & 52 & 53 & 54 & 55 & 56 \\
\hline & $662-670$ & $670-678$ & $678-686$ & 686-694 & 694-702 & $702-710$ & 710-718 & 718-726 & $726-734$ & $734-742$ & $742-750$ & $750-758$ \\
\hline & 57 & 58 & 59 & 60 & 61 & 62 & 63 & 64 & 65 & 66 & 67 & 68 \\
\hline & $758-766$ & $766-774$ & $774-782$ & $782-790$ & 790-798 & 798-806 & $806-814$ & $814-822$ & $822-830$ & $830-838$ & $838-846$ & $846-854$ \\
\hline & 69 & & & & & & & & & & & \\
\hline & 854-862 & & & & & & & & & & & \\
\hline & & & $\begin{array}{l}\text { Retai } \\
\text { interle }\end{array}$ & $\begin{array}{l}\mathrm{d} / \\
\mathrm{ed}\end{array}$ & & & $\begin{array}{l}\text { Clear } \\
\text { spect }\end{array}$ & & & & PMSE & \\
\hline
\end{tabular}

Figure 2 470-862 MHz TV "white space" spectrum [41].

wind and solar powered base station on the Isle of Bute, and a $6 \mathrm{~m}$ high hybrid wind and solar powered base station recently installed on the Isle of Tiree.

On the Isle of Bute a renewable powered "WindFi" base station has been operating over 24 months with different radio payloads to assess the performance of the renewable power system. Outdoor tests on Bute using "white space" test-kit based on a modified WiFi radio with a 5 $\mathrm{MHz}$ bandwidth (operating in an $8 \mathrm{MHz}$ channel) have demonstrated Transmission Control Protocol (TCP) throughput up to $3 \mathrm{Mbit} / \mathrm{s}$ in a $5 \mathrm{MHz}$ channel for a 4.8 $\mathrm{km}$ non-LOS link in a UHF channel at $2 \mathrm{~W}$ EIRP, and higher than $10 \mathrm{Mbit} / \mathrm{s}$ for $2 \mathrm{~km}$ connections. Further trials are now ongoing under the auspices of a UK Government Technology Strategy Board Grant in collaboration with British Telecom PLC, BBC, Steepest Ascent Ltd, Netpropagate Ltd and Berg Design Ltd which commenced in April 2011 and will run until September 2012.

Trials now running on Tiree currently involve 20 subscribers served by the renewable powered base station to replace a legacy wireless network that has over 100 subscribers. Initial results have demonstrated longest link TCP throughputs of up to $80 \mathrm{Mbit} / \mathrm{s}$ over a $9 \mathrm{~km} 5$ $\mathrm{GHz}$ band C PTP link and up to $20 \mathrm{Mbit} / \mathrm{s}$ over a $3 \mathrm{~km}$ LOS, $5 \mathrm{GHz}$ band B PTMP link. Further expansion of the Tiree trial network to be completed by March 2012 is planned with three more renewable base station deployments, five mains powered base stations, and redundancy planning to create a ring of eight base stations serving all subscribers and providing visitor access.

\subsection{Community or cooperative operating models}

In addition to technological issues and the challenge of finding good backhaul connectivity, it is also worth noting that a good operating and business model is required. One potential operating and business model is that of a community or cooperatively owned network [29]. Operating in locations and environments that are uneconomical and have no return on investment for larger companies may be possible with managed community cooperatives. For example infrastructure and ISP services could be owned by the community and operated by a network manager who will set the tariffs and access charges in consultation with a cooperative or community type trust (as already operates on Tiree). Besides network coverage, the network can serve as framework to test and develop network support for important evolving applications such as smart grid communications [30] and real time services such as $\mathrm{BBC}$ iPlayer for video streaming. As such the network on Tiree can operate to provide connectivity to homes connected on the HopScotch network, and also provide a platform to further test and develop; in particular for "white space" radio given the potential of the beachfront UHF spectrum available in many rural areas.

\section{3 "WindFi" base station}

Each HopScotch wireless base station is an ultra-low power autonomous unit, powered by a combination of wind and solar renewable sources as shown in Figure 4. Using renewable energy allows each base station to 


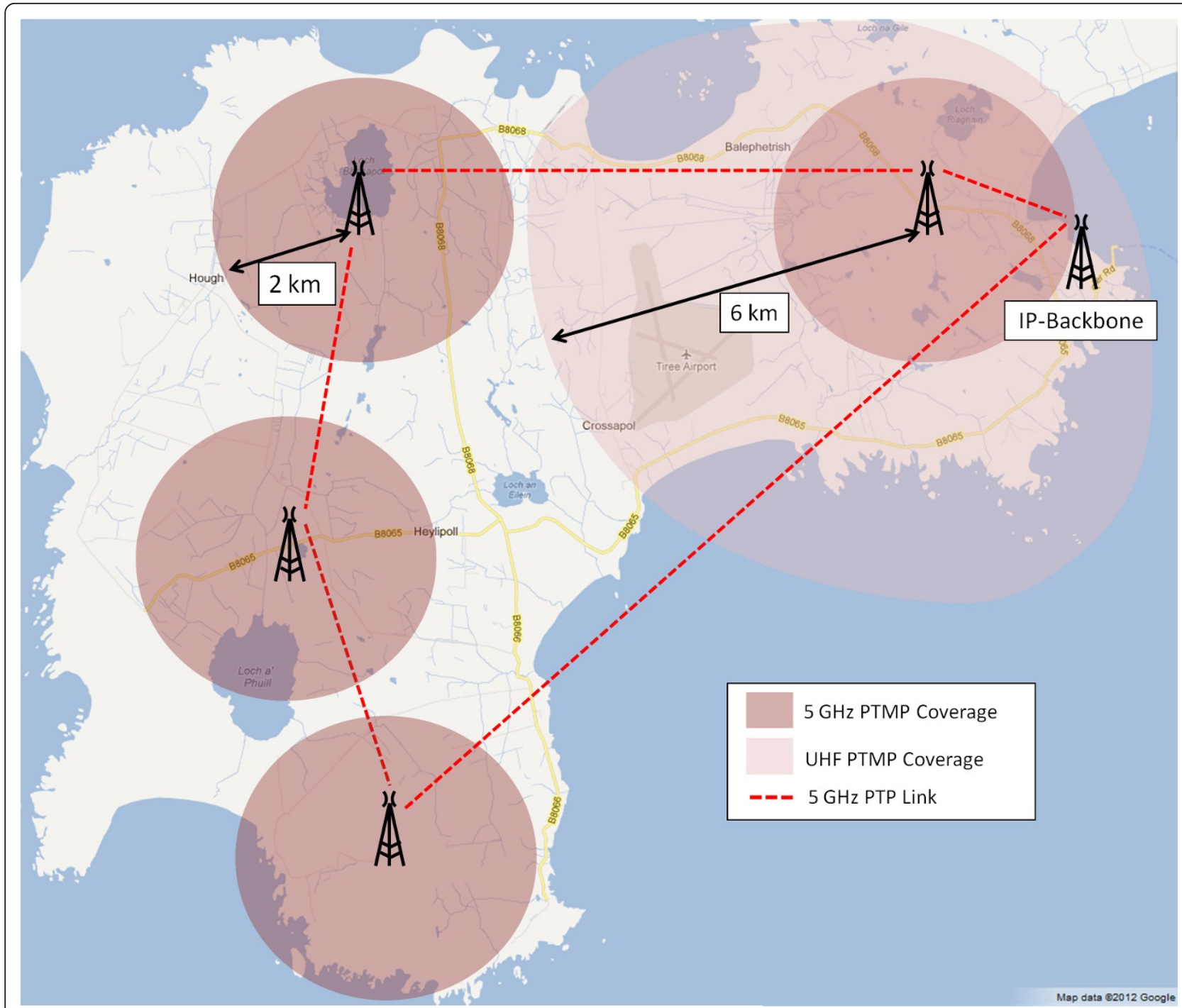

Figure 3 Loop network connecting several communities to the IP-backbone via PTP and PTMP links.

operate independently of fixed electrical infrastructure, allowing for flexible, optimised placement. A base station requires no permanent foundations and therefore requires no specific planning permission in the UK to install in most scenarios. The base station in Figure 4 was installed by 2 persons and is mounted on a tripod bolted on to wooden posts in the ground. This base station has to date survived $90 \mathrm{mph}$ winds.

\subsection{Base station subsystems}

The base station electronics are divided into two subsystems-the renewable power system and the radio payload-which are discussed below.

A HopScotch "WindFi" base station is designed to handle different radio payloads, providing flexibility to best suit a community's needs. A typical deployment to serve a medium sized community would provide four radio sectors for PTMP user access and two PTP links for back-haul, requiring a total of six radios. The radios are managed by two single board computers (SBC). The emergence of ultra low voltage yet computationally powerful processors such as the Intel Atom and ARM based embedded processors allow a single processor to manage multiple radio interfaces on a modest power budget. Each SBC operates two PTMP radios and one PTP radio by running a software MAC for each radio and performing packet routing within the mast and network. The use of two SBCs combined with the ring network topology creates redundancy within the base station. With each of the two PTP links connecting the base station to the IP-backbone in clock- or anti-clockwise direction within the ring topology, if one SBC fails 


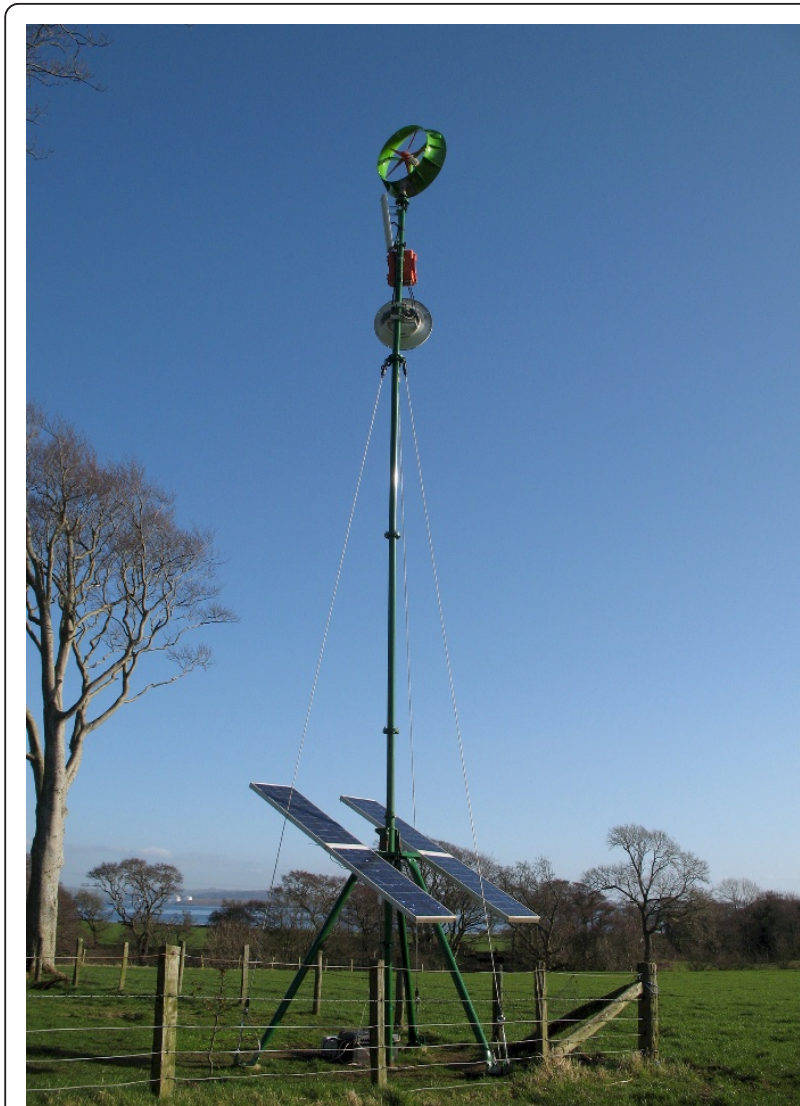

Figure 4 Prototype "WindFi" base station on the Isle of Bute, Scotland, which has been running since early 2010 . The base contains solar panels and batteries, over which a $10 \mathrm{~m}$ mast carries the wind turbine with antennas and radio equipment directly beneath.

the second SBC will still be operational. This remaining PTP link can provide back-haul for the PTMP radios connected to the unaffected SBC.

To minimize transmission losses between the radios and antennas, the SBCs with attached radios are placed as close to the antennas as possible, allowing the transmit power to be minimised to maintain a desired EIRP.

A battery bank is used to store energy generated by the wind turbine and PV modules and acts as an energy source for the system. A hybrid web-connected charge controller manages charging to prolong battery life and provides resistive loads for the generation units when production is too great. The current status of the wind turbine, PV array, battery bank and loads are monitored remotely in real-time to allow problems to be detected early and corrective action to be taken, minimizing base station downtime. A web interface allows the PV panels to be remotely positioned to facilitate solar tracking and maximise the generation potential of the PV array.

\subsection{Power system sizing}

For deployment in Scotland, HopScotch uses both wind turbines and PV modules to harvest a combination of wind and solar energy. As discussed in [11], wind and solar energy sources are complementary in Scotland; in the summer months solar energy peaks whilst in the winter months winds prevail. Figure 5 shows the average daily energy which can be harvested from an $80 \mathrm{~W}$ PV module and a $200 \mathrm{~W}$ wind turbine on the Isle of Tiree.

Due to the high cost of PV modules, wind turbines and batteries, a substantial amount of research has focused on sizing solar and wind sources and battery banks for given loads and conditions [11,12,31,32]. The battery bank in a "WindFi" system is sized to allow for continuous operation without any additional energy input for $N_{\mathrm{d}}$ days and without the capacity dropping below the maximum discharge depth $D \%$ to prolong the battery lifespan. The required battery bank capacity $C_{\mathrm{b}}$ in $\mathrm{kWh}$ for a daily energy demand $E_{\mathrm{d}}$ in $\mathrm{kWh}$ is given by

$$
C_{\mathrm{b}}=\frac{N_{\mathrm{d}} E_{\mathrm{d}}}{D} .
$$

Two SBCs are used in each base station for redundancy creating a load of up to $10 \mathrm{~W}$. Assuming each radio requires up to $5 \mathrm{~W}$ the total power requirement of six radios is up to $30 \mathrm{~W}$. Due to the additional power requirements for the mast power system, monitoring and control, antenna motorisation and the inclusion of a safety margin, a base load of $50 \mathrm{~W}$ is used for sizing the power system, creating a maximum daily energy demand of $E_{\mathrm{d}}=1.2 \mathrm{kWh}$ assuming a $24 \mathrm{~h}$ operation under full load.

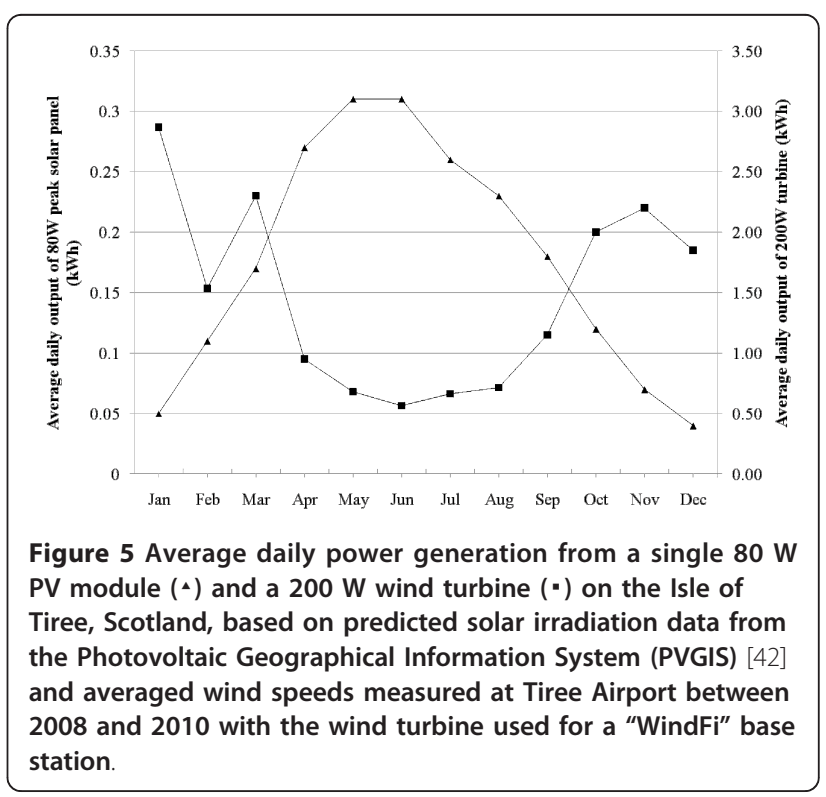


In our design the system must remain operational for $N_{\mathrm{d}}=3$ days without any energy input and without the stored energy dropping below $D=50 \%$ of the total capacity. This mitigates against overcast, wind free days and protects the batteries from over-discharge. Therefore the battery bank must be able to store $C_{\mathrm{b}}=7.2$ $\mathrm{kWh}$, leading to a required battery bank capacity of $600 \mathrm{Ah}$ when utilising $12 \mathrm{~V}$ rated batteries.

The energy production of the mast must be capable of recharging the batteries from the lowest allowed charge depth in $N_{\mathrm{r}}$ days in addition to maintaining the base load. The required energy production per day $E_{\mathrm{p}}$ required to recharge the battery bank over $N_{\mathrm{r}}$ days is

$$
E_{\mathrm{p}}=E_{\mathrm{d}}+E_{\mathrm{r}}=E_{\mathrm{d}}+\frac{D C_{\mathrm{b}}}{N_{\mathrm{r}}} .
$$

Therefore the total energy production required per day, $E_{\mathrm{p}}$, is the base load energy demand $E_{\mathrm{d}}$ plus the recharge energy demand $E_{\mathrm{r}}$. When allowing $N_{\mathrm{r}}=5$ days to recover from a maximum lost charge, an extra energy demand of $E_{\mathrm{r}}=720 \mathrm{Wh}$ is required, creating a total daily demand of $E_{\mathrm{p}}=1.92 \mathrm{kWh}$.

In the Highlands and Islands of Scotland the potential of wind energy is greater than that of PV. In the winter months the mean wind turbine output of around $2 \mathrm{kWh}$ per day is sufficient to meet our requirement, while in the summer months the output drops to around 600 Wh per day. To meet the system's energy demand of $E_{\mathrm{p}}$ $=1.92 \mathrm{kWh}$, the PV must be capable of contributing 1.3 $\mathrm{kWh}$ per day assuming an optimal orientation given by PVGIS, therefore requiring around six $80 \mathrm{~W}$ PV units according to the characterisation in Figure 5.

\subsection{Solar tracking}

Solar tracking increases the output of solar panels by aligning the solar panel with the current position of the sun. Generally three types of solar tracking are used:

- inclined tracking rotates the panel around the north/south axis at a fixed inclination;

- vertical tracking rotates the panel around a vertical axis at a fixed inclination;

- two-axis tracking allows the panel to rotate and incline.

The gain in efficiency of using the above three types of solar tracking on the Isle of Tiree is provided in Figure 6. During the summer months vertical axis tracking provides roughly a $35 \%$ increase in energy taken per day. Therefore with vertical solar tracking, only $0.8 \mathrm{kWh}$ of rated generation potential are required, which allows to reduce the amount of PV units from six to only four. Although two-axis tracking offers the largest efficiency

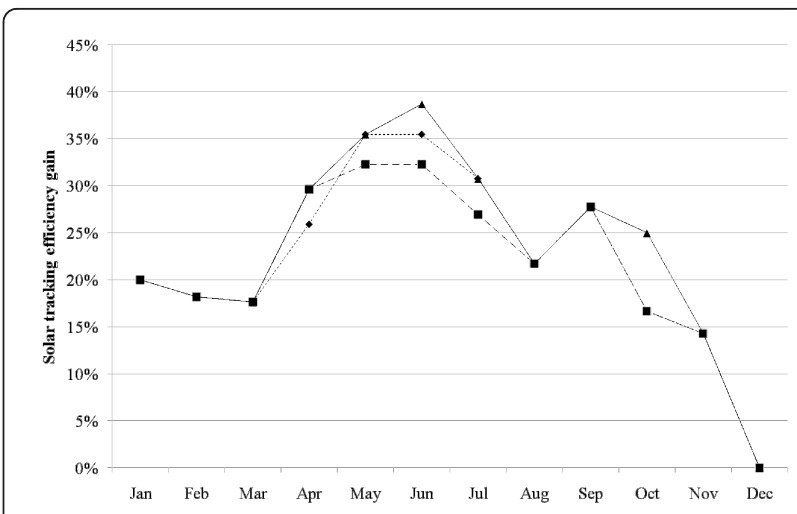

Figure 6 Average gain in energy generation per day using vertical $(\diamond)$, inclined $(\cdot)$ and two-axis $(\bullet)$ solar tracking over a fixed installation on the Isle of Tiree using PVGIS

gains, the modest efficiency improvement over single axis tracking, added complexity for motorisation in two planes and the increased risk of failure make it an undesirable solution.

Motorising the solar panels incurs an additional energy requirement of around 5 Wh per day, but the added efficiency more than compensates for this penalty. The resulting reduction in the number of PV modules also reduces stresses on the mechanical structure through decreased wind loading, which can be further eased by feathering motorised PV panels during strong winds.

\section{Optimising spectrum usage}

While most wireless rural broadband access systems rely on WiFi technologies in the $2.4 \mathrm{GHz}$ and $5 \mathrm{GHz}$ bands, HopScotch utilises a combination of $5 \mathrm{GHz}$ WiFi and UHF frequency bands for transmission. Therefore, below we analyse how the use of UHF TVWS bands can reduce the burden on base station coverage and transmit power requirements, compared to transmission at $5 \mathrm{GHz}$.

\subsection{Advantages of "white space"}

Wireless networks transmitting in the TVWS band have been estimated to cover four times the area that can be reached via current unlicensed bands in the $2.4 \mathrm{GHz}$ and $5 \mathrm{GHz}$ region, thus reducing the number of base stations required [33]. This can be attributed to the relatively benign propagation characteristics of TVWS frequencies [34]. Conversely, when covering the same area, operation in TVWS frequencies as compared to transmission in the $5 \mathrm{GHz}$ band allows to lower the transmit power in the downlink and achieves better SNR in the uplink. Sections 4.1.1, 4.1.2, and 4.1.3 discuss several factors which contribute to this performance which are 
relevant to rural areas. Section 4.2 analyses how the use of UHF frequencies affects the required number of base stations and link transmission power for an example community.

\subsubsection{Free space path loss}

The transmission loss over a distance $r$ is frequency dependent as the effective antenna aperture of a fixed gain antenna decreases with increasing frequency. When operating at two different transmission frequencies $f_{1}$ and $f_{2}$ for isotropic antennas with identical gains Frii's transmission equation [35] can be rearranged to

$$
r_{\left(f_{1}\right)}=\left(\frac{f_{2}}{f_{1}}\right) r_{\left(f_{2}\right)}
$$

in order to relate the distances $r_{\left(f_{i}\right)}, i=1,2$, over which an equivalent loss is experienced. Thus, given a fixed receive signal level, the propagation range at a lower frequency is greater for a lower frequency than a higher frequency [36]. According to (3), at $630 \mathrm{MHz}$ (the middle frequency of the TVWS band) range is increased 9 times compared to $5.67 \mathrm{GHz}$ (the middle frequency of $5 \mathrm{GHz}$ bands B and C). Similarly the transmit powers required to receive the same power at the same distance when transmitting at frequencies $f_{\dot{v}} i=\{1,2\}$ relate by

$$
P_{t_{\left(f_{1}\right)}}=\left(\frac{f_{2}}{f_{1}}\right)^{2} P_{t_{\left(f_{2}\right)}}
$$

i.e. to transmit at $630 \mathrm{MHz}$ requires $19.1 \mathrm{~dB}$ less power than transmitting at $5.67 \mathrm{GHz}$ under the constraint of identical distances and receive powers.

\subsubsection{Terrain effects and diffraction loss}

Point to point propagation path loss can be predicted under obstructive, non-LOS conditions between base station and terminal. As the size of the obstruction is much larger than the wavelength of the radio wave, knife-edge diffraction can be used to estimate the shadow loss [37]. The propagation loss $L_{\mathrm{ke}}$ due to knife edge diffraction as sketched in Figure 7 can be estimated using the Fresnel diffraction parameter $v$. A good approximation is given by

$$
v \approx-h_{p} \sqrt{\frac{2\left(r_{1}+r_{2}\right)}{\left(\lambda r_{1} r_{2}\right)}}
$$

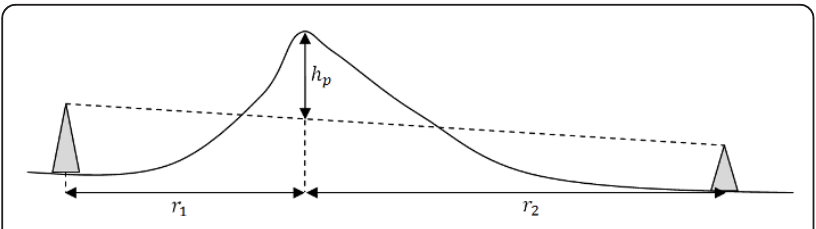

Figure 7 Knife edge diffraction parameters with $h_{p}$ the height difference between the virtual LOS between the transmit and receive antennas and the peak of the obstruction. The quantities $r_{1}$ and $r_{2}$ are measures of the distances between the edge, and the transmitter and receiver, respectively, as outlined in Figure 7. Based on the wavelength $\lambda$ of the carrier frequency, the propagation loss $L_{k e}$ can then be calculated using Fresnel integrals [35].

As an example, over a $5 \mathrm{~km}$ link with a $30 \mathrm{~m}$ knife edge obstruction $900 \mathrm{~m}$ from the transmitter, the diffraction loss at $5.67 \mathrm{GHz}$ is $9.4 \mathrm{~dB}$ higher than the estimated loss of $20.1 \mathrm{~dB}$ at $630 \mathrm{MHz}$.

\subsubsection{Foliage}

Clutter such as vegetation, which can be common in rural environments, affects the propagation of radio waves. Studies have shown the attenuation to dependent on both frequency and polarisation of the wave. A greater loss is experienced at a vertical than a horizontal polarisation due to the existence of large vertical components in the vegetation medium [38] up to about 1 $\mathrm{GHz}$ [39]. Weissberger's model predicts the propagation loss due to the presence of trees in a point to point link [40], with a path loss $L_{w}$

$$
L_{w}= \begin{cases}0.45(f)^{0.284}(d), & \text { for } 0 \leq d \leq 14 \\ 1.33(f)^{0.284}(d)^{0.588}, & \text { for } 14<d \leq 400\end{cases}
$$

for a given frequency $f[\mathrm{GHz}]$ and a depth $d[\mathrm{~m}]$ of foliage along the path.

As an example, using Weissberger's model for a foliage depth of $10 \mathrm{~m}$ the estimated propagation loss of 7.4 $\mathrm{dB}$ due to the foliage obstruction at $5.67 \mathrm{GHz}$ is $3.4 \mathrm{~dB}$ higher than the loss experiences at $630 \mathrm{MHz}$.

\subsection{Network design considerations}

Using TVWS frequencies provides wider coverage in rural areas compared to the $5 \mathrm{GHz}$ band due to the smaller losses discussed above. This allows households situated further away from the community hub to be reached using fewer base stations or a lower transmit power.

\subsubsection{Base station placement}

An example community of six households (labelled A to F) for HopScotch is shown in Figure 8. The optimum base station placement to serve this community can be determined using the Radio Mobile planning tool for a base station height of $10 \mathrm{~m}$ and a maximum permitted transmit power of $1 \mathrm{~W}$ EIRP for $5 \mathrm{GHz}$ band $\mathrm{B}$ transmissions. Radio Mobile uses the Longley-Rice propagation model for non-LOS links and the two-ray path model for LOS links [35]. The effects of foliage and other clutter have been ignored for this study but will be the subject of a future study using geographic information system (GIS) data. When using $5 \mathrm{GHz}$ band $\mathrm{B}$, 


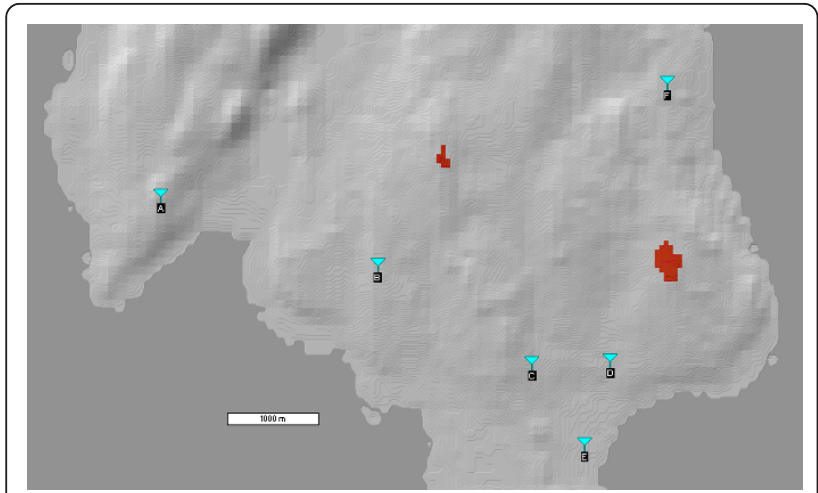

Figure 8 Example community with areas for a potential base station to provide coverage at $630 \mathrm{MHz}$ at $1 \mathrm{~W}$ EIRP.

no single base station can cover all six nodes given a minimum received signal strength of $-85 \mathrm{dBm}$, the minimum receive signal strength observed during trial tests to maintain a reasonable connection. At TVWS frequencies $(630 \mathrm{MHz}$ for this analysis) two locations allow coverage of all six nodes as shown by the red shading in Figure 8.

Whilst no single base station using $5 \mathrm{GHz}$ bands can serve the community at $1 \mathrm{~W}$ EIRP, coverage for the entire community can be achieved by introducing two communicating base stations as shown in Figure 9. Base station A (BSA) can serve nodes $\mathrm{C}, \mathrm{D}, \mathrm{E}$ and $\mathrm{F}$ with omnidirectional coverage and base station $\mathrm{B}$ (BSB) can serve nodes A and B.

Using a combination of $5 \mathrm{GHz}$ and UHF frequencies for this scenario allows the available data rate to be maximized for each user using only one base station. Base station A (BSA) can serve users C, D, E, and F at 5 $\mathrm{GHz}$, providing a greater bandwidth and hence data rate for users. A white space overlay on BSA also allows it to

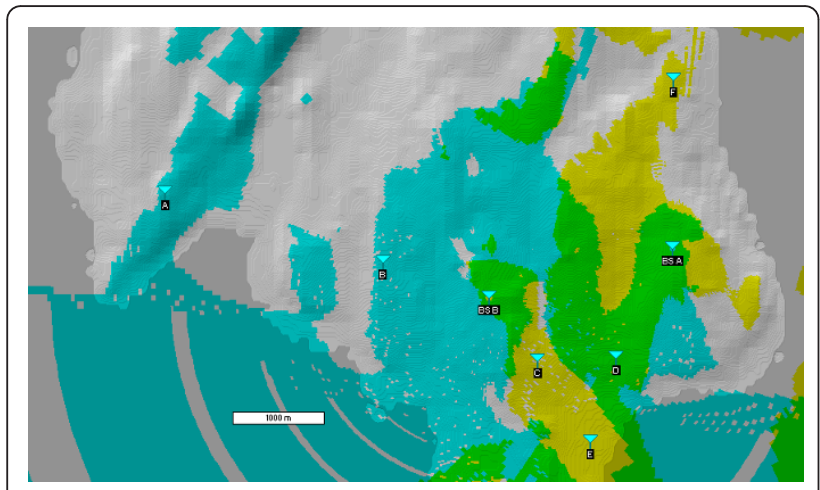

Figure 9 Coverage provided by two base stations at $5660 \mathrm{MHz}$ $1 \mathrm{~W}$ EIRP, with yellow shading representing coverage provided by base station $A(B S A)$, blue shading representing the coverage provided by base station B (BSB), and the overlapping coverage indicated by green shading service users A and B without the need for an additional base station.

\subsubsection{Link transmission power}

An alternative to adding additional base stations at 5 $\mathrm{GHz}$ to cover all nodes is to increase the transmit power above $1 \mathrm{~W}$ EIRP. This may be possible in some regulatory environments. To demonstrate the required transmission power, two links are considered between base station BSA and users A and D. The elevation profiles of two links are shown in Figures 10 and 11. The link between BSA and user A is $5.6 \mathrm{~km}$ long and is non-LOS. The link between BSA and user D is $1.35 \mathrm{~km}$ long and contains no obstructions. The expected received signal power $P_{R x}$ in decibels for a given transmission power $P_{T x}$, transmit and receive antenna gains $\left(G_{T x}, G_{R x}\right)$ line losses $\left(L_{T \infty} L_{R x}\right)$ and path loss $L_{P L}$ is given by

$$
P_{R x}=P_{T x}+G_{T X}-L_{T X}-L_{P L}+G_{R x}-L_{R x} .
$$

Similarly for a given receive power the required transmit power in decibels can be calculated

$$
P_{T x}=P_{R x}-G_{T x}+L_{T x}+L_{P L}-G_{R x}+L_{R x} .
$$

Using (7) and (8), Table 2 shows the simulated path loss between base station BSA and users A and D and the expected receive power given a transmit and receive antenna gain of $14 \mathrm{dBi}$, line losses of $0.5 \mathrm{~dB}$ and an EIRP of $1 \mathrm{~W}$. The required transmission power to create a link with a received signal strength of $-85 \mathrm{dBm}$ is also calculated. To create a link between base station BSA and user A, a substantial EIRP and therefore transmission power would be required in the $5 \mathrm{GHz}$ band which is not permitted in the UK and is detrimental for a renewable powered system due to increased power consumption.

Table 2 also contains expected link performance at UHF frequencies using the same system parameters. A
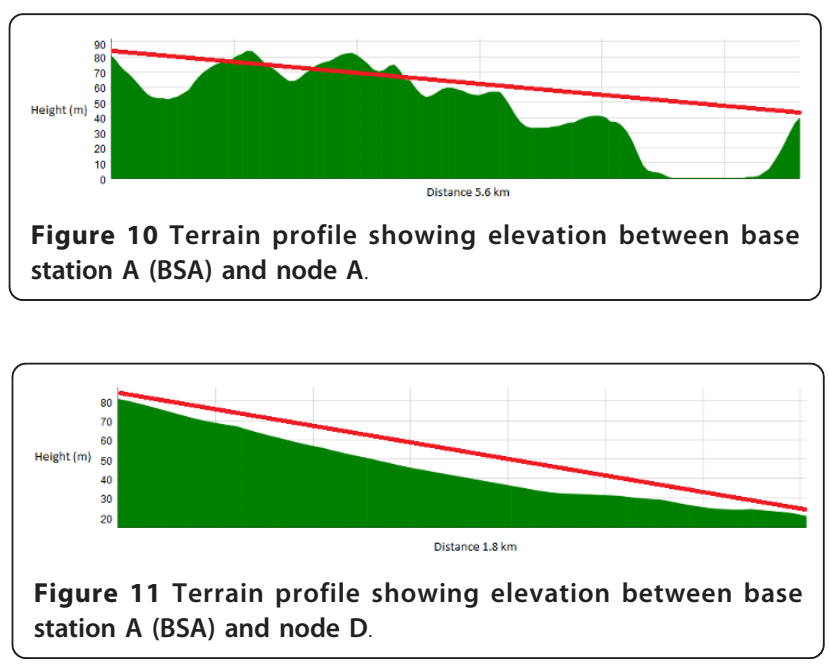
Table 2 Link calculations and transmit and receive powers between the base station and nodes A and D

\begin{tabular}{lllll}
\hline Node & A @ $\mathbf{6 3 0} \mathbf{~ M H z}$ & A @ $\mathbf{5 6 6 0 ~} \mathbf{~ H H z}$ & D @ $\mathbf{6 3 0} \mathbf{~ M H z}$ & D @ 5660 $\mathbf{~ M H z}$ \\
\hline Distance & $5.60 \mathrm{~km}$ & $5.60 \mathrm{~km}$ & $1.35 \mathrm{~km}$ & $1.35 \mathrm{~km}$ \\
Path Loss & $117.8 \mathrm{~dB}$ & $144.6 \mathrm{~dB}$ & $97.2 \mathrm{~dB}$ & $114.6 \mathrm{~dB}$ \\
Rx Level @ 1 W EIRP & $-74.3 \mathrm{dBm}$ & $-101.1 \mathrm{dBm}$ & $-53.7 \mathrm{dBm}$ & $-71.1 \mathrm{dBm}$ \\
Tx power @ -85.0 dBm & $5.8 \mathrm{dBm}$ & $32.6 \mathrm{dBm}$ & $-14.8 \mathrm{dBm}$ & $2.6 \mathrm{dBm}$ \\
\hline
\end{tabular}

substantial reduction in path loss is expected at UHF frequencies compared to $5 \mathrm{GHz}$, especially in the longer non-LOS link. Therefore when using a UHF link, the propagation characteristics allow a reduction in transmit power or an increase in receive power compared to the $5 \mathrm{GHz}$ band, reducing power consumption in the power amplifier or improving user throughputs by allowing higher order modulation schemes and code rate to be used. As each link is fixed the transmission power can be set during installation using channel measurements to achieve the desired receive signal strength.

\section{Conclusion}

This article has discussed how a combination of green radio techniques enables the provision of a low-cost rural broadband solution through a network infrastructure referred to as HopScotch. Low-power and low-cost WiFi based radio equipment allows renewable energy "WindFi" base stations to be operated by renewable sources, reducing operating costs, fuel use, and eliminating the requirement of access to the electricity grid. Additionally, "WindFi" base stations are capable of transmitting via an overlay using TV "white space" frequency bands. In this article, we have particularly focussed on some of the green aspects of "WindFi" systems; relying on a low power, renewable design and the use of UHF frequency bands, where additional transmit power savings can contribute to the efficiency of the system.

We have demonstrated that the propagation characteristics of UHF frequencies in TV "white space" bands can result in fewer base stations being required to illuminate a community or the required transmission power can be reduced compared to operation in Gigahertz frequency bands. The dimensioning of the system is based on successful initial field results, with a further expansion of trials on the Isles of Bute and Tiree planned.

\section{Acknowledgements}

The authors wish to acknowledge support and contributions British Telecom PLC, the BBC, Berg Design Ltd, Netpropagate Ltd, Steepest Ascent Ltd, and the UK Government Technology Strategy Board (TSB) grant for the Extending Broadband Reach Project supporting the Isle of Bute white space testbed. The authors would like to further acknowledge support from the Scottish Funding Council (SFC), Highlands and Islands Enterprise (HIE), a Strathclyde University EPSRC/British Telecom Knowledge Transfer Account
(KTA) Grant, the Engineering and Physical Sciences Research Council (EPSRC), and the Co-Operative Enterprise Fund for various aspects of study presented in this article.

\section{Author details}

${ }^{1}$ Centre for White Space Communications, Department of Electronic \& Electrical Engineering, University of Strathclyde, Glasgow G1 1XW, Scotland, UK ${ }^{2}$ Steepest Ascent Ltd., 94 Duke Street, Glasgow G4 OUW, Scotland, UK

\section{Competing interests}

The authors declare that they have no competing interests.

Received: 2 October 2011 Accepted: 16 March 2012

Published: 16 March 2012

\section{References}

1. R Wolff, E Andrews, Broadband access, citizen enfranchisement, and telecommunications services in rural and remote areas: a report from the American frontier. IEEE Commun Mag. 48(5), 128-135 (2010)

2. Radio Systems for Rural Communications. in IEE Colloquium, vol. 018. (London, UK, 1993). IEE Professional Group E8

3. L Vishloff, M Callendar, A Winter, Wireless telecommunications technologies for rural applications, in IEEE International Conference on Personal Wireless Communications, Bangalore, India, pp. 26-31 (1994)

4. P Chitamu, S Braithwaite, Non-coherent detection of narrow-band modulation for use in an advanced rural radio-telephone network, in IEE Colloquium on Radio Systems for Rural Communications, vol. 018. London, UK, pp. 3/1-3/4 (1993)

5. J Mosenthal, B Nleya, N Manthoko, Broadband/future generation network services deployment in rural and remote areas, in 2nd International Conference on Adaptive Science Technology, Accra, Ghana), pp. 128-132 (2009)

6. J Riding, J Ellershaw, A Tran, L Guan, T Smith, Economics of broadband access technologies for rural areas, in Conference on Optical Fiber Communication, San Diego, CA, pp. 1-3 (2009)

7. D Žagar, V Križanović, Analyses and comparisons of technologies for rural broadband implementation, in 17th International Conference on Software, Telecommunications Computer Networks, Hvar, pp. 292-296 (2009)

8. M Zhang, R Wolff, Crossing the digital divide: cost-effective broadband wireless access for rural and remote areas. IEEE Commun Mag. 42(2), 99-105 (2004)

9. W Gerok, S Rusche, P Unger, Hybrid broadband access with IEEE 802.16e: an economic approach for rural areas, in IEEE Mobile WiMAX Symposium, Napa Valley, CA, pp. 93-97 (2009)

10. YC Liang, AT Hoang, HH Chen, Cognitive radio on TV bands: a new approach to provide wireless connectivity for rural areas. IEEE Wirel Commun. 15(3), 16-22 (2008)

11. G Bernardi, P Buneman, MK Marina, Tegola tiered mesh network testbed in rural Scotland, in Proceedings of the 2008 ACM workshop on Wireless networks and systems for developing regions, ACM, New York, USA, pp. 9-16 (2008)

12. A Farbod, $\mathrm{T}$ Todd, Resource allocation and outage control for solarpowered WLAN mesh networks. IEEE Trans Mobile Comput. 6(8), 960-970 (2007)

13. D Wu, D Gupta, P Mohapatra, Quail Ridge Wireless Mesh Network: Experiences, Challenges and Findings, in 3rd International Conference on Testbeds and Research Infrastructure for the Development of Networks and Communities Orlando, USA, pp. 1-6 (2007)

14. B Raman, K Chebrolu, Experiences in using WiFi for rural Internet in India. IEEE Communications Magazine. 45(1), 104-110 (2007) 
15. P Bhagwat, B Raman, D Sanghi, SIGCOMM, (Karlsruhe, Germany, 2003), pp. 33-38. Turning 802.11 inside-out

16. T Todd, A Sayegh, M Smadi, D Zhao, The need for access point power saving in solar powered WLAN mesh networks. IEEE Netw. 22(3), 4-10 (2008)

17. N Michael, AP Vinod, C Moy, J Palicot, Design of multistandard channelization accelerators for software defined radio handsets. IEEE Trans Signal Process. 59(10), 4767-4780 (2011)

18. T Chen, H Zhang, Z Zhao, X Chen, Towards green wireless access networks, in 5th International ICST Conference on Communications and Networking in China Beijing, China, pp. 1-6 (2010)

19. X Chen, Z Zhao, H Zhang, Green transmit power assignment for cognitive radio networks by applying multi-agent Q-learning approach, in European Wireless Technology Conference Paris, France, pp. 113-116 (2010)

20. I Krikidis, J Thompson, S McLaughlin, P Grant, Feedback based relaying in long term evolution systems. International Conference on Wireless Communications and Signal Processing Suzhou, China 1-5 (2010)

21. C Comaniciu, N Mandayam, H Poor, Radio resource management for Green wireless networks. 70th IEEE Vehicular Technology Conference Fall Anchorage, USA 1-5 (2009)

22. J Palicot, Cognitive radio: an enabling technology for the green radio communications concept, in Proceedings of the 2009 International Conference on Wireless Communications and Mobile Computing: Connecting the World Wirelessly ACM, New York, USA, pp. 489-494 (2009)

23. J He, P Loskot, T O'Farrell, V Friderikos, S Armour, J Thompson, Energy efficient architectures and techniques for Green Radio access networks, in 5th International ICST Conference on Communications and Networking in China Beijing, China, pp. 1-6 (2010)

24. D Grace, T Chen, $T$ Jiang, P Mitchell, Using cognitive radio to deliver 'Green' communications, in 4th International Conference on Cognitive Radio Oriented Wireless Networks and Communications Hannover, Germany, pp. 1-6 (2009)

25. R Mangharam, R Rajkumar, S Pollin, F Catthoor, B Bougard, L Van der Perre, I Moeman, Optimal fixed and scalable energy management for wireless networks, in Proceedings on IEEE INFOCOM 2005, vol. 1. (Miami, USA, 2005), pp. $114-125$

26. R Min, M Bhardwaj, M Ickes, A Wang, AP Chandrakasan, The hardware and the network: total-system strategies for power aware wireless microsensors, in 2002 CAS Workshop on Wireless Communication and Networking, (Pasadena, USA., 2002) http://descanso.jpl.nasa.gov/Symposia/ieee_cas2002/ full/min.pdf

27. A Payne, Implementation challenges for UHF white space cognitive systems, in IET Seminar on Cognitive Radio and Software Defined Radios: Technologies and Techniques, London, UK, pp. 1-30 (2008)

28. Federal Communications Commission: Unlicensed Operation in the TV Broadcast Bands. Federal Register. 74, 7314-7332 (2009)

29. M Manshaei, P Marbach, JP Hubaux, Evolution and market share of wireless community networks, in International Conference on Game Theory for Networks Istanbul, Turkey, pp. 508-514 (2009)

30. M Brew, F Darbari, L Crockett, M Fitch, S Weiss, R Stewart, UHF White Space Network for Rural Smart Grid Communication, in Proceedings IEEE Smart Grid Communications Conference Brussels, pp. 138-142 (2011)

31. S Hashimoto, T Yachi, T Tani, A new stand-alone hybrid power system with wind generator and photovoltaic modules for a radio base station, in 26th Annual International Telecommunications Energy Conference Chicago, USA, pp. 254-259 (2004)

32. B Panajotovic, B Odadzic, Design and "intelligent" control of hybrid power system in telecommunication, in 15th IEEE Mediterranean Electrotechnical Conference Valletta, Malta, pp. 1453-1458 (2010)

33. B Lennett, Rural broadband and the TV white space how unlicensed access to vacant television channels can bring affordable wireless broadband to rural America, in New America Foundation Wireless Future Program. 22 http://www.newamerica.net/files/nafmigration/ Rural_Broadband_and_TV_WhiteSpace.pdf (2008)

34. D Borth, R Ekl, B Oberlies, S Overby, Considerations for successful cognitive radio systems in US TV white space, in 3rd IEEE Symposium on New Frontiers in Dynamic Spectrum Access Networks, Chicago, USA, pp. 1-5 (2008)

35. S Saunders, A Arag'on-Zavala, Antennas and Propagation for Wireless Communication Systems, (John Wiley \& Sons Ltd, Chichester, England, 2007)

36. S Srikanteswara, D Choudhury, A review of TV Whitespace portable devices, in 2010 IEEE Radio and Wireless Symposium, New Orleans, USA, pp. 480-483 (2010)
37. WC Lee, Mobile Communications Engineering, (McGraw-Hill Professional, New York, 1997)

38. AR Tharek, Radio wave propagation studies through tropical Vegetation in Malaysia at VHF, in Proceedings of ISAP, Sapporo, Japan, pp. 897-900 (1992)

39. Attenuation in Vegetation (1994). ITU: Recommendation ITU-R PN.833-1

40. KL Chee, SA Torrico, T Kurner, Foliage attenuation over mixed terrains in rural areas for broadband wireless access at $3.5 \mathrm{GHz}$. IEEE Trans Antennas Propag. 59(7), 2698-2706 (2011)

41. Consultation on licence-exempting cognitive devices using interleaved spectrum. Tech rep (2009). OFCOM: Digital dividend: cognitive access

42. Photovoltaic Geographical Information System (PVGIS) http://re.jrc.ec.europa. eu/pvgis/ (2008)

doi:10.1186/1687-1499-2012-112

Cite this article as: McGuire et al:: HopScotch-a low-power renewable energy base station network for rural broadband access. EURASIP Journal on Wireless Communications and Networking 2012 2012:112.

\section{Submit your manuscript to a SpringerOpen ${ }^{\mathcal{O}}$ journal and benefit from:}

- Convenient online submission

- Rigorous peer review

- Immediate publication on acceptance

- Open access: articles freely available online

- High visibility within the field

- Retaining the copyright to your article

Submit your next manuscript at $\gg$ springeropen.com 